\title{
Effect of DA-9701 on the Normal Motility and Clonidine-induced Hypomotility of the Gastric Antrum in Rats
}

\author{
Je Wook Kang, ${ }^{1}$ Dae Kyeong Han, ${ }^{2}$ Ock Nyun Kim, ${ }^{2}$ and Kwang Jae Lee ${ }^{1 *}$ \\ ${ }^{1}$ Department of Gastroenterology and ${ }^{2}$ Laboratory Animal Research Center, Ajou University School of Medicine, Suwon, Gyeonggi-do, Korea
}

\section{Background/Aims}

DA-9701 is a novel prokinetic agent. In the present study, we investigated the effect of DA-9701 on the motility of the gastric antrum in the normal and clonidine-induced hypomotility in an in vivo animal model.

\section{Methods}

A strain gauge force transducer was sutured on the gastric antrum to measure the contractile activity in rats. A total of 28 rats were subclassified into the 4 groups: (1) the placebo group, (2) the DA-9701 group, (3) the placebo group in the clonidine-pretreated rats, and (4) the DA-9701 group in the clonidine-pretreated rats. After the basal recording, either placebo (3\% [w/v] hydroxypropylmethyl cellulose) or DA-9701 was administered. Contractile signals were measured after the administration and after a meal. In the clonidinepretreated rats, either placebo or DA-9701 was administered. Contractile signals were measured after the administration and after a meal.

\section{Results}

Oral administration of DA-9701 did not significantly alter the motility index of the gastric antrum in the preprandial and postprandial periods, compared with the placebo group. The administration of clonidine decreased the motility index of the gastric antrum in the preprandial and postprandial periods, compared with the administration of placebo. This reduction of the antral motility by the administration of clonidine was not observed in the clonidine-pretreated DA-9701 group. The percentage of the motility index in the postprandial period was significantly greater in the clonidine-pretreated DA-9701 group, compared with the clonidine-pretreated placebo group.

\section{Conclusions}

DA-9701 improves the hypomotility of the gastric antrum induced by clonidine, suggesting its gastroprokinetic effect in the pathologic condition.

(J Neurogastroenterol Motil 2016;22:304-309)

\section{Key Words}

Antrum; DA-9701; Motility; Prokinetic

\footnotetext{
Received: August 13, 2015 Revised: December 6, 2015 Accepted: December 20, 2015

(a) This is an Open Access article distributed under the terms of the Creative Commons Attribution Non-Commercial License (http://creativecommons. org/licenses/by-nc/4.0) which permits unrestricted non-commercial use, distribution, and reproduction in any medium, provided the original work is properly cited.

*Correspondence: Kwang Jae Lee, MD, PhD Department of Gastroenterology, Ajou University Hospital, Ajou University School of Medicine, 164 Worldcup-ro, Yeongtong-gu, Suwon, Gyeonggi-do 443-721, Korea Tel: +82-31-219-5102, Fax: +82-31-219-5999, E-mail: kjleemd@hotmail.com
} 


\section{Introduction}

Functional dyspepsia (FD) is characterized by the presence of recurrent or persistent symptoms thought to originate in the gastroduodenal region without any organic, systemic or metabolic disease that is likely to explain the symptoms. ${ }^{1}$ Diverse pathophysiologic mechanisms are known to be involved in FD. Delayed gastric emptying has been proposed to be an important pathophysiologic mechanism of FD. ${ }^{2,3}$ The term prokinetic refers to a group of medications accelerating gastrointestinal motility. The pathophysiologic mechanism related to the effectiveness of prokinetics in FD is considered to be mainly delayed gastric emptying. Delayed gastric emptying is observed in approximately $30-40 \%$ of patients with FD. ${ }^{4,5}$ Actually, prokinetic drugs are commonly prescribed for the treatment of dyspeptic patients suspected to have delayed gastric emptying. However, currently available gastroprokinetic agents have some demerits such as adverse effects or conflicting efficacy data. $^{6-8}$

DA-9701 is a novel prokinetic agent formulated with the extracts of Pharbitis Semen and Corydalis Tuber. These plants have been used in oriental traditional medicine. Pharbitis Semen, the seed of Pharbitis nil Choisy, is believed to have analgesic effects. Corydalis Tuber, the root of Corydalis yahusuo W.T. Wang (Papaveraceae), is known to have analgesic and anti-ulcer effects. ${ }^{9-11}$ DA-9701 was reported to not only significantly enhance gastric emptying in healthy rats, but also normalize delayed gastric emptying induced by apomorphine, cisplatin or stress. ${ }^{12,13}$ These findings suggest that DA-9701 may become a new therapeutic option for the treatment of patients with delayed gastric emptying. However, the effect of DA-9701 on the normal motility and hypomotility, a pathologic condition, and of the gastric antrum, has yet to be fully investigated.

Thus, in the present study, we aimed to investigate the effect of DA-9701 on the motility of the gastric antrum in the normal and clonidine-induced hypomotility in an in vivo animal model.

\section{Materials and Methods}

\section{Preparation of DA-9701}

DA-9701, the standardized extract of Pharbitis Semen and Corydalis Tuber, was prepared as previously reported. ${ }^{14}$ Briefly, dried herbs (water content of $<10 \%$ ) were mixed in a specific ratio of the 2 herbs and extracted with $50 \%$ aqueous ethanol at room tem- perature for 48 hours. After filtration, the aqueous ethanol extract was evaporated under reduced pressure to yield a brown extract. The quality was evaluated using the standard method involving quantitative high-performance liquid chromatography, as previously reported. ${ }^{12}$

\section{Animals}

Male Sprague-Dawley rats aged 6 weeks were purchased and housed in individual cages under controlled conditions of temperature $\left(22-24^{\circ} \mathrm{C}\right)$ and illumination (12-hour light cycle starting at 6 AM) for at least 1 week. The rats were given ad libitum access to food and water. The rats underwent surgical procedure to place the strain gauge force transducer, and were subsequently allowed at least 1 week to acclimate to that condition. All the experiments were performed in 28 rats $(n=7$ in each group) aged 8 weeks (200-250 g). The experimental procedures were performed in accordance with the guidelines of the Institutional Animal Care and Use Committee of Ajou University. All efforts were made to minimize animal suffering and to reduce the number of animals in experiments.

\section{Measurement of Gastric Antral Motility in Conscious Rats}

After fasting for 24 hours, the rats were anesthetized with intraperitoneal administration of Zoletil (tiletamine + zolazepam, 0.06 $\mathrm{mL} / 100 \mathrm{~g}$ ) and Rompun (xylazine, $0.04 \mathrm{~mL} / 100 \mathrm{~g}$ ). A midline incision was made in the abdomen under anesthesia, and the stomach was exteriorized. Strain gauge transducers (F-061S; Star Medical, Inc, Tokyo, Japan) were sutured onto the serosal surface of the gastric antrum to measure the contractile activity of the circular muscle. The abdomen was then closed, and the wires from transducer were exteriorized through the abdominal wall, ran under the skin toward the back. Wires were protected by a protective jacket (Star Medical, Inc, Tokyo, Japan). After the surgery, rats were housed individually and were allowed to recover for 1 week before the experiments. On the experimental day, the wires from the transducer were connected to the recording system (Power-Lab model 4SP; ADI instruments, Colorado Springs, CO, USA), and motility recording was started at a constant time in the morning.

\section{Study Protocols for the Effect of DA-9701 on the Normal Motility of the Antrum}

After the basal recording for 20 minutes in the fasting state, either placebo (3\% [w/v] hydroxypropylmethyl cellulose) (Sigma, St. Louis, MO, USA) or DA-9701 (3 mg/kg) was administered orally. Contractile signals were measured for 20 minutes from 30 minutes 
after the administration (the preprandial period). Subsequently, an oropharyngeal feeding tube was inserted, and then the standardized liquid meal was administered through the feeding tube. The recording continued for 20 minutes after the oral infusion of the meal (the postprandial period) (Fig. 1A).

\section{Study Protocols for the Effect of DA-9701 on the Clonidine-induced Hypomotility}

After the basal recording for 20 minutes in the fasting state, clonidine hydrochloride (clonidine), an $\alpha 2$-adrenoceptor agonist, at a dose of $100 \mu \mathrm{g} / \mathrm{kg}$ was subcutaneously administered. The contractile signals were measured for 20 minutes after the administration of clonidine (after clonidine). Subsequently, either placebo (3\% [w/v] hydroxypropylmethyl cellulose) or DA-9701 (3 mg/kg) was administered orally. After 30 minutes, the contractile signals were recorded for 20 minutes (the preprandial period). Subsequently, oropharyngeal feeding tube was inserted, and then the standardized liquid meal was administered through the feeding tube. The recording continued for 20 minutes after the oral infusion of the meal (the postprandial period) (Fig. 1B).

\section{Statistical Methods}

All data are expressed as the mean \pm standard deviation. Motility index and the area under the curve was calculated using a computer-assisted system (PowerLab, ADInstruments, Colorado Springs, CO, USA). The motility index was determined as the area under the curve (AUC) in the antrum for a 20-minute period, and is shown as a percentage ( $\%$ motility index $=100 \times$ [AUC posttreatment/AUC pretreatment or basal period]). These percentages were compared between groups using Student's $t$ test, and among groups using one-way ANOVA with multiple comparisons. Statistical analyses were performed using SPSS version 18.0 (IBM Corp, Armonk, NY, USA). A P-value of less than 0.05 was considered to be statistically significant.

\section{Results}

\section{Effects of Placebo or DA-9701 on the Normal Motility of the Antrum}

Oral administration of DA-9701 did not significantly alter the motility index of the gastric antrum in the preprandial $(P=0.675)$ and postprandial periods $(P=0.632)$, compared with the placebo group (Fig. 2A and 2B) (Table 1).

\section{Effects of Clonidine on the Antral Motility of the Stomach}

The administration of clonidine decreased the motility index of the gastric antrum in the preprandial $(P=0.053)$ and postprandial periods $(P=0.017)$, compared with the administration of placebo (Fig. 2C and Table 2).

\section{Effects of DA-9701 on the Clonidine-induced Hypomotility}

In the clonidine-pretreated DA-9701 group, the reduction of the antral motility by the administration of clonidine was not observed in the preprandial $(P=0.601)$ and postprandial periods $(P$ $=0.673)$, compared with the placebo group. The percentage motility index in the postprandial period was significantly greater in the clonidine-pretreated DA-9701 group, compared with the clonidine-

A

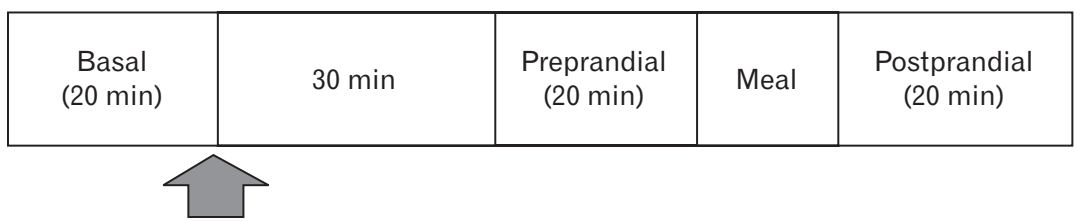

Placebo or DA-9701

B

\begin{tabular}{|c|c|c|c|c|c|}
\hline $\begin{array}{c}\text { Basal } \\
(20 \mathrm{~min})\end{array}$ & $\begin{array}{c}\text { After } \\
\text { clonidine } \\
\text { (20 min) }\end{array}$ & $30 \mathrm{~min}$ & $\begin{array}{c}\text { Preprandial } \\
\text { (20 min) }\end{array}$ & Meal & $\begin{array}{l}\text { Postprandial } \\
\text { (20 min) }\end{array}$ \\
\hline
\end{tabular}

Figure 1. Study protocols. (A) After the basal recording for 20 minutes, either placebo $(3 \%[\mathrm{w} / \mathrm{v}]$ hydroxypropylmethyl cellulose) or DA-9701 (3 mg/kg) was administered orally, and then the standardized liquid meal was administered through the feeding tube. (B) After the basal recording for 20 minutes, clonidine hydrochloride (clonidine) at a dose of $100 \mu \mathrm{g} / \mathrm{kg}$ was subcutaneously administered. Subsequently, either placebo (3\% $[\mathrm{w} / \mathrm{v}]$ hydroxypropylmethyl cellulose) or DA-9701 was administered orally, and then the standardized liquid meal was administered through the feeding tube. 
A

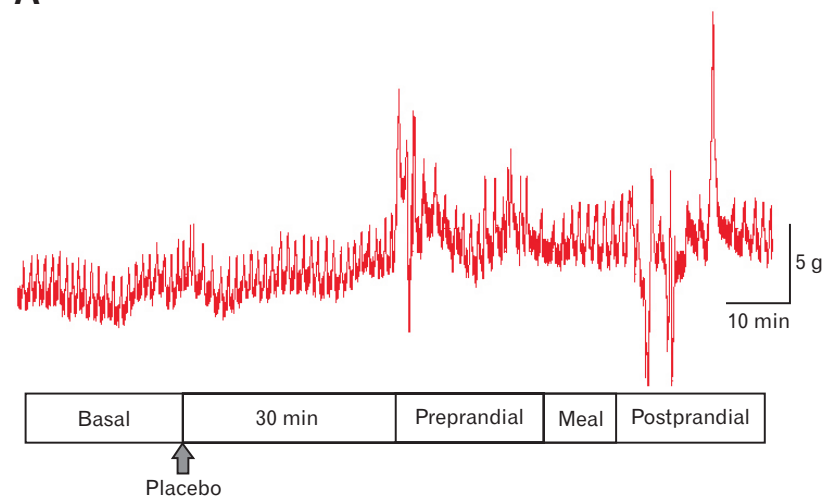

C

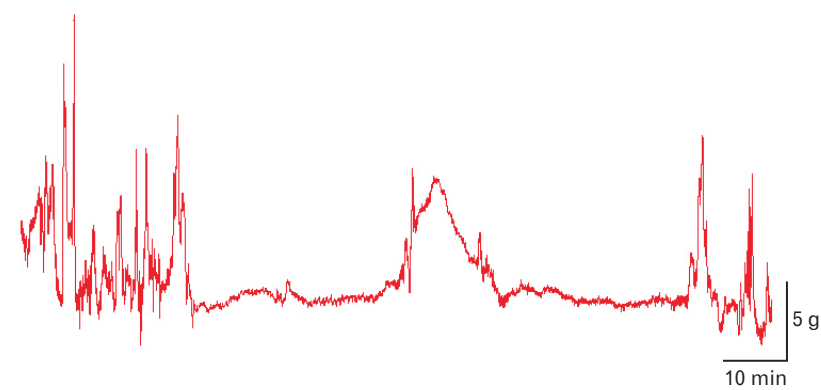

B
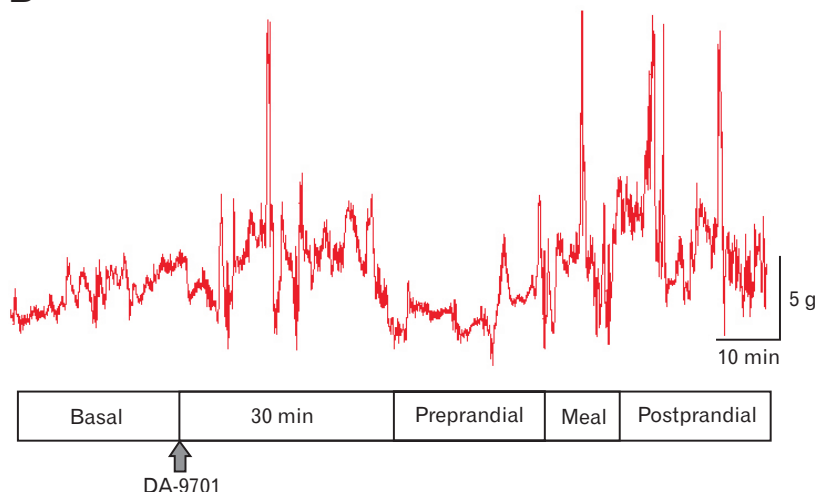

D

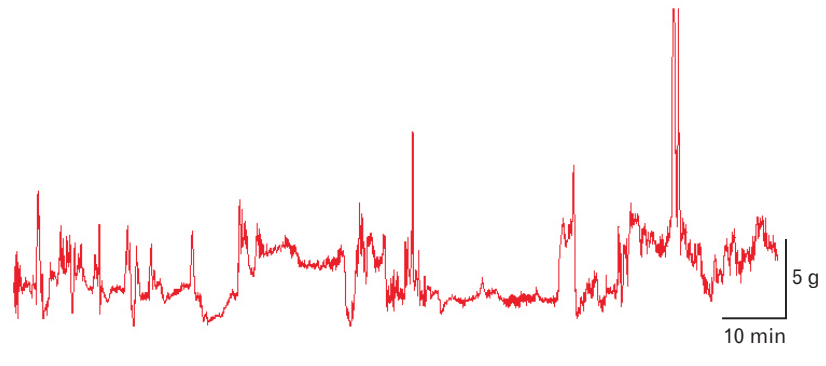

Figure 2. Effects of placebo or DA-9701 on normal motility and hypomotility of the gastric antrum. Compared with the placebo group (A), the motility index of the gastric antrum was not significantly changed after the administration of DA-9701 (B), both in the preprandial and postprandial periods. In the clonidine-pretreated placebo group, the motility index of the gastric antrum was significantly reduced, both in the preprandial and postprandial periods (C), but this hypomotility was not observed in the clonidine-pretreated DA-9701 group (D).

Table 1. Effects of Placebo or DA-9701 on the Normal Motility of the Gastric Antrum

\begin{tabular}{lcc}
\hline & \multicolumn{2}{c}{$\%$ motility index compared to the basal period } \\
\cline { 2 - 3 } & Preprandial period & Postprandial period \\
\hline Placebo & $112.5 \pm 36.2$ & $127.9 \pm 40.1$ \\
DA-9701 & $125.2 \pm 62.5$ & $142.7 \pm 60.9$ \\
$P$-value & 0.675 & 0.632 \\
\hline
\end{tabular}

pretreated placebo group $(P=0.041)$ (Fig. 2D and Table 2).

\section{Discussion}

DA-9701 is a currently available prokinetic agent in South Korea, named Motilitone (Dong-A ST, Seoul, South Korea). This agent is more like a herbal drug rather than a chemical drug, because it is formulated with the extracts of Pharbitis Semen and Co-
Table 2. Effects of DA-9701 on the Clonidine-induced Hypomotility

\begin{tabular}{lcc}
\hline & $\%$ motility index compared to the basal period \\
\cline { 2 - 3 } & Preprandial period & Postprandial period \\
\hline Placebo & $112.5 \pm 36.2$ & $127.9 \pm 40.1$ \\
Clonidine + placebo & $65.8 \pm 24.3^{\mathrm{a}}$ & $63.5 \pm 37.9^{\mathrm{b}}$ \\
Clonidine + DA-9701 & $100.4 \pm 19.9$ & $117.4 \pm 23.1^{\mathrm{c}}$ \\
\hline
\end{tabular}

${ }^{a} P=0.05$, compared with the placebo group.

${ }^{\mathrm{b}} P<0.05$, compared with the placebo group.

${ }^{c} P<0.05$, compared with the clonidine + placebo group.

rydalis Tuber. The present study demonstrated that DA-9701 improved clonidine-induced hypomotility of the gastric antrum. This finding suggests a beneficial role of DA-9701 in the treatment of hypomotility of the gastric antrum observed in a subset of patients with FD.

A recent study in rats demonstrated that DA-9701 improved 
stress-induced delay in gastric emptying. ${ }^{13}$ In addition, animal studies have shown the beneficial effect of DA-9701 on gastric accommodation and visceral sensitivity. ${ }^{14,15}$ Since DA-9701 is a herbal compound formulated with Pharbitidis Semen and Corydalis Tuber, it has an affinity to multiple receptors associated with gastroduodenal function. ${ }^{16}$ This agent is suggested to be involved in dopamine $\mathrm{D} 2$, serotonin receptor $4\left(5-\mathrm{HT}_{4}\right), 5-\mathrm{HT}_{1 \mathrm{~A}}$, and adrenergic $\alpha 2$ receptors. Its action on dopamine $\mathrm{D}_{2}$ and $5-\mathrm{HT}_{4}$ receptors explains the prokinetic effect of DA-9701. This mechanism appears to be important in enhancing upper gastrointestinal motility. Moreover, the affinity of DA-9701 to $5-\mathrm{HT}_{1 \mathrm{~A}}$ and adrenergic $\alpha 2$ receptors is likely to be associated with the fundus relaxing effect of this drug. A phase III study in patients with FD demonstrated the comparable effect of DA-9701 to itopride. DA-9701 showed noninferior efficacy to itopride that is known to increase acetylcholine concentrations by inhibiting dopamine $\mathrm{D}_{2}$ receptors and acetylcholinesterase. ${ }^{17}$ Actually, itopride was reported to stimulate gastric motility and accelerate gastric emptying. ${ }^{18}$ In several Asian countries such as South Korea and Japan, mosapride citrate (mosapride), a serotonin $5-\mathrm{HT}_{4}$ receptor agonist, as well as itopride has been used in patients with $\mathrm{FD}$ as a prokinetic drug. A recent animal study showed the comparable effect of DA-9701 to mosapride in reversing delayed gastric emptying induced by acute stress. ${ }^{13}$ However, itopride and mosapride have shown variable efficacy in the treatment of FD, particularly in placebo-controlled randomized studies. ${ }^{19,20}$ To date, there is no report regarding a placebo controlled trial of DA-9701 in patients with FD. Therefore, the efficacy of DA-9701 in FD needs to be confirmed.

Delayed gastric emptying has been reported to be a major pathophysiologic mechanism of $\mathrm{FD}^{2-4}$ Gastric antral hypomotility is suggested to contribute to delayed gastric emptying. ${ }^{21}$ Clonidine, an adrenergic $\alpha 2$ receptor agonist, is known to induce hypomotility and delayed transit of the stomach and intestine, via the inhibition of acetylcholine release from cholinergic nerve terminals. ${ }^{22,23}$ A delayed gastric emptying model induced by $\alpha 2$-adrenoceptor agonists has been used to demonstrate the effect of gastroprokinetic agents in animals. ${ }^{24,25}$ In the present study, we showed that the pretreatment of clonidine significantly reduced motility of the gastric antrum, that is keeping with the previous observations. ${ }^{26,27}$ Hypomotility was observed both in the preprandial and postprandial periods. The gastroprokinetic activity of prokinetics may be different among experimental models of gastric dysfunction. Given that the administration of DA-9701 recovered antral hypomotility induced by clonidine, the prokinetic effect of DA-9701 through dopamine $\mathrm{D}_{2}$ and $5-\mathrm{HT}_{4}$ receptors might be stronger than its relaxing effect via adrenergic $\alpha 2$ receptors.

In the present study, the effect of DA-9701 on the normal motility was not remarkable. No significant change in the motility index of the gastric antrum was observed in the preprandial and postprandial periods, compared with the placebo group. This may indicate that it does not increase motility in patients with normal motility of the gastric antrum. The reason for that cannot be explained exactly. However, it might be attributed to the feature of a herbal drug. In addition, the probability of a type II error cannot be excluded. In contrast to the negative effect of DA-9701 in the normal condition, its effect on clonidine-induced hypomotility, a pathologic condition, was distinct. The recovery of antral hypomotility by DA-9701 may result in improvement of delayed gastric emptying. Therefore, our results suggest that DA-9701 is a potent prokinetic agent that can be used for the treatment of gastric hypomotility. However, studies revealing the prokinetic effect of DA-9701 in humans are lacking. Thus, further studies to elucidate the beneficial effect of DA-9701 on the pathophysiologic mechanisms of FD are required. Since this agent is a herbal extract, no serious adverse events related to DA9701 have been reported in clinical settings. Therefore, DA-9701 appears to be a promising prokinetic agent for the treatment of FD.

The present study has several limitations. The number of rats included in the experiments may not be appropriate for the analysis. Therefore, the possibility of type II errors in the results cannot be completely excluded. In addition, the artifacts produced by motion, tension, stress, operations, manipulations, etc. might not be completely removed in the analysis due to technical limitations.

In conclusion, DA-9701 improves the hypomotility of gastric antrum induced by clonidine. Our results suggest that the prokinetic activity of DA-9701 may improve delayed gastric emptying or meal-related symptoms of FD. Placebo-controlled randomized trials of DA-9701 in patients with postprandial distress syndrome are expected. In addition, the effects of DA-9701 on the gastric emptying and accommodation to a meal in humans need to be studied.

Financial support: This study was financially supported by Dong-A ST.

\section{Conflicts of interest: None.}

Author contributions: Je Wook Kang acquired data and analyzed data; Dae Kyeong Han and Ock Nyun Kim performed experiments and acquired data; and Kwang Jae Lee made the concept of the study, analyzed data, wrote the paper, and revised the manuscript. 


\section{References}

1. Tack J, Talley NJ, Camilleri M, et al. Functional gastroduodenal disorders. Gastroenterology 2006;130:1466-1479.

2. Stanghellini V, Tosetti C, Paternico A, et al. Risk indicators of delayed gastric emptying of solids in patients with functional dyspepsia. Gastroenterology 1996;110:1036-1042.

3. Sarnelli G, Caenepeel P, Geypens B, Janssens J, Tack J. Symptoms associated with impaired gastric emptying of solids and liquids in functional dyspepsia. Am J Gastroenterol 2003;98:783-788.

4. Tack J, Lee KJ. Pathophysiology and treatment of functional dyspepsia. J Clin Gastroenterol 2005;39:S211-S216.

5. Lee KJ, Kindt S, Tack J. Pathophysiology of functional dyspepsia. Best Pract Res Clin Gastroenterol 2004;18:707-716.

6. Tack J, Camilleri M, Chang L et al. Systematic review: cardiovascular safety profile of 5- $\mathrm{HT}_{4}$ agonists developed for gastrointestinal disorders. Aliment Pharmacol Ther 2012;35:745-767.

7. Holtmann G, Talley NJ, Liebregts T, Adam B, Parow C. A placebo-controlled trial of itopride in functional dyspepsia. N Engl J Med 2006;354: 832-840.

8. Hallerbäck BI, Bommelaer G, Bredberg E et al. Dose finding study of mosapride in functional dyspepsia: a placebo-controlled, randomized study. Aliment Pharmacol Ther 2002;16:959-967.

9. Lee TH, Son M, Kim SY. Effects of corydaline from Corydalis tuber on gastric motor function in an animal model. Biol Pharm Bull 2010;33: 958-962.

10. Hung TM, Na M, Dat NT et al. Cholinesterase inhibitory and antiamnesic activity of alkaloids from Corydalis turtschaninovii. J Ethnopharmacol 2008;119:74-80.

11. Hung TM, Ngoc TM, Youn UJ, et al. Anti-amnestic activity of pseudocoptisine from Corydalis Tuber. Biol Pharm Bul 2008;31:159-162.

12. Lee TH, Choi JJ, Kim DH, et al. Gastroprokinetic effects of DA-9701, a new prokinetic agent formulated with Pharbitis Semen and Corydalis Tuber. Phytomedicine 2008;15:836-843.

13. Jung YS, Kim MY, Lee HS, Park SL, Lee KJ. Effect of DA-9701, a novel prokinetic agent, on stress-induced delayed gastric emptying and hormonal changes in rats. Neurogastroenterol Motil 2013;25:254-259.

14. Kim ER, Min BH, Lee TH, Son M, Rhee PL. Effect of DA-9701 on colorectal distension-induced visceral hypersensitivity in a rat model. Gut Liver 2014;8:388-393.

15. Kim ER, Min BH, Lee SO, Lee TH, Son M, Rhee PL. Effects of
DA-9701, a novel prokinetic agent, on gastric accommodation in conscious dogs. J Gastroenterol Hepatol 2012;27:766-772.

16. Kwon YS, Son M. DA-9701: a new multi-acting drug for the treatment of functional dyspepsia. Biomol Ther 2013;21:181-189.

17. Choi MG, Rhee PL, Park H, et al. Randomized, controlled, multicenter trial: comparing the safety and efficacy of DA-9701 and itopride hydrochloride in patients with functional dyspepsia. J Neurogastroenterol Motil 2015;21:414-422.

18. Huang X, Lv B, Zhang S, Fan YH, Meng LN. Itopride therapy for functional dyspepsia: a meta-analysis. World J Gastroenterol 2012;18: 7371-7377.

19. Hallerbäck BI, Bommelaer G, Bredberg E, et al. Dose finding study of mosapride in functional dyspepsia: a placebo-controlled, randomized study. Aliment Pharmacol Ther 2002;16:959-967.

20. Talley NJ, Tack J, Ptak T, Gupta R, Giguère M. Itopride in functional dyspepsia: results of two phase III multicentre, randomised, doubleblind, placebo-controlled trials. Gut 2008;57:740-746.

21. Kusunoki H, Haruma K, Hata J, et al. Real-time ultrasonographic assessment of antroduodenal motility after ingestion of solid and liquid meals by patients with functional dyspepsia. J Gastroenterol Hepatol 2000;15:1022-1027.

22. Colucci R, Blandizzi C, Carignani D, Placanica G, Lazzeri G, Del Tacca M. Effects of imidazoline derivatives on cholinergic motility in guineapig ileum: involvement of presynaptic $\alpha 2$-adrenoceptors or imidazoline receptors? Naunyn Schmiedebergs Arch Pharmacol 1998;357:682-691.

23. Asai T, Mapleson WW, Power I. Differential effects of clonidine and dexmedetomidine on gastric emptying and gastrointestinal transit in the rat. Br J Anaesth 1997;78:301-307.

24. García-Garayoa E, Monge A, Roca J, Del Río J, Lasheras B. VB20B7, a novel 5-HT-ergic agent with gastrokinetic activity. II. Evaluation of the gastroprokinetic activity in rats and dogs. J Pharm Pharmacol 1997;49: 66-73.

25. Tanaka T, Mizumoto A, Mochiki E, Suzuki H, Itoh Z, Omura S, Effects of EM574 and cisapride on gastric contractile and emptying activity in normal and drug-induced gastroparesis in dogs. J Pharmacol Exp Ther 1998;287:712-719.

26. Kawachi M, Matsunaga Y, Tanaka T, et al. Acotiamide hydrochloride (Z-338) enhances gastric motility and emptying by inhibiting acetylcholinesterase activity in rats. Eur J Pharmacol 2011;666:218-225.

27. Fülöp K, Zádori Z, Rónai AZ, Gyires K. Characterisation of $\alpha 2$ adrenoceptor subtypes involved in gastric emptying, gastric motility and gastric mucosal defense. Eur J Pharmacol 2005;528:150-157. 